\title{
Parallel electric field structures associated with the low-frequency oscillations in the auroral plasma
}

\author{
R. V. Reddy ${ }^{1}$, S. V. Singh ${ }^{1}$, G. S. Lakhina ${ }^{1}$, and R. Bharuthram ${ }^{2}$ \\ ${ }^{1}$ Indian Institute of Geomagnetism, New Panvel, Navi Mumbai-410218, India \\ ${ }^{2}$ University of Witwatersrand, Johannesburg 2000, South Africa
}

(Received December 12, 2005; Revised July 3, 2006; Accepted July 4, 2006; Online published September 29, 2006)

\begin{abstract}
The nonlinear evolution of low-frequency electrostatic oscillations in a magnetized plasma consisting of protons, electrons and oxygen ion beams has been studied. The fluid equations have been used for the oxygen beam, whereas the Boltzmann distributions are used for the protons and electrons. The coupled system of equations are reduced to a single nonlinear differential equation in the rest frame of the propagating wave for any direction of propagation with respect to the ambient magnetic field. This nonlinear differential equation is solved numerically for the parameters charateristic of the auroral acceleration region. Depending on the wave Mach number, proton and oxygen ion concentrations, and driving electric field, the numerical solutions show a range of periodic solutions varying from sinusoidal to sawtooth and highly spiky waveforms. The effects of the plasma parameters, in particular the oxygen ion concentration and the proton temperature on the evolution of the nonlinear waves are examined. The results from the model are compared with satellite observations.
\end{abstract}

Key words: Auroral plasma, electric fields, low frequency waves.

\section{Introduction}

The electrostatic ion cyclotron (EIC) waves have been studied extensively in the auroral plasma because of their important role in heating and acceleration of the plasma (Kelley et al., 1975). The EIC waves are found to be unstable to current-driven instabilities in the auroral region (Kindel and Kennel, 1971). The measurements performed by the S3-3 (Mozer et al., 1977), Viking (Andre et al., 1987), Polar (Mozer et al., 1997) and FAST (Ergun et al., 1998; Cattell et al., 1998) satellites have shown that EIC waves are frequently observed in the auroral magnetosphere at altitudes between $3000 \mathrm{~km}$ to $8000 \mathrm{~km}$ and beyond.

The nonlinear electrostatic ion cyclotron waves propagating nearly perpendicular to the magnetic field have been studied by Temerin et al. (1979). In their model, spiky structures were observed in the perpendicular electric field for large Mach numbers.

Polar (Mozer et al., 1997) and FAST (Ergun et al., 1998) satellite obeservations in the auroral magnetosphere have shown that the nonlinear, time domain structures associated with the parallel electric field exhibit spiky wave forms. The Polar data (Mozer et al., 1997) also showed that the perpendicular electric field exhibits spiky structures with amplitudes about $400 \mathrm{mV} / \mathrm{m}$ with repetition rates in the range of local ion cyclotron frequency. The FAST (Ergun et al., 1998) satellite observations revealed large amplitude parallel electric fields associated with ion cyclotron waves with an ion beam in the upward current region, whereas in the downward current region, it showed spiky electric field

Copyright (c) The Society of Geomagnetism and Earth, Planetary and Space Sciences (SGEPSS); The Seismological Society of Japan; The Volcanological Society of Japan; The Geodetic Society of Japan; The Japanese Society for Planetary Sciences; TERRAPUB structures in both perpendicular and parallel components. The perpendicular electric field structures represent ion cyclotron waves at a frequency $f \sim 200 \mathrm{~Hz}$, the time series of the parallel electric field reveal a spiky waveform at a lower frequency (see figure 6 of Ergun et al., 1998). These quasi-static, parallel electric field structures are thought to be responsible for particle acceleration in the auroral acceleration region.

Cattell et al. (1998) have reported observations of waves near the cyclotron frequencies of $\mathrm{H}^{+}, \mathrm{O}^{+}$and $\mathrm{He}^{+}$and the associated ion beams and field-aligned currents during nightside auroral zone crossing of FAST. The waves below as well as near the oxygen ion cyclotron frequencies were large amplitude and with non-sinusoidal wave forms.

Recenlty, using the fluid theory, Reddy et al. (2002) have studied nonlinear low frequency electric field structures in a two-component magnetized plasma consisting of cold ions and warm electrons. It is found that for sufficiently large values of the driver electric field, the governing equation representing the electric field, produced by nonlinear coupling between the ion acoustic mode and ion cyclotron oscillations, led to the generation of sawtooth and highly spiky periodic waveforms. The periods of these waveforms range from ion cyclotron to ion acoustic.

Bharuthram et al. (2002) have studied the low-frequency nonlinear waves in the auroral plasma consisting of electrons with Boltzmann distribution and warm ions. It is found that the finite ion temperature supresses the nonlinearity in the ion cyclotron wave due to enhanced dispersive effects and decreases the periodicity of the oscillations. Whereas the periodicity of the ion acoustic wave is enhanced with increasing ion temperature. Following the approach of Reddy et al. (2002), Moolla et al. (2003) stud- 
ied high-frequency nonlinear waves in the Earth's magnetosphere consisting of hot electrons, cold electrons and cold ions. They have found that the hot electrons were accelerated in bursts leading to rapid changes in their speeds.

The low-frequency oscillations in a plasma with spatially variable field-aligned flow has been studied by Ganguli et al. (2002). They have found that a transverse velocity gradient in the parallel ion flow can generate multiple cyclotron harmonic waves and the resulting electric field structures are found to be spiky in nature and the peaks are separated by an ion cyclotron period. Kim et al. (2005) have studied the generation of spiky electric field structures associated with multi-harmonic electrostatic ion cyclotron waves in a double ended Q machine. They have observed that a linear combination of a multi-harmonic spectrum of electrostatic ion cyclotron waves when the harmonics have comparable amplitudes and phase-locked could lead to the generation of spiky electric potential waveforms.

In this paper, we study the low-frequency nonlinear waves in a multi-component auroral plasma consisting of oxygen ion beam and Boltzmann distributions for electrons and protons. In Section 2, we present the model and the basic equations. The numerical results and discussion are presented in Section 3. Finally, the conclusions and the relevance of our work to the auroral ionosphere is discussed in Section 4.

\section{Model}

We consider a homogeneous magnetized threecomponent plasma consisting of protons, cold oxygen ion beam drifting along the magnetic field direction with speed $v_{0}$, and electrons. The finite amplitude ioncyclotron/ion-acoustic waves are propagating in the $x$ direction at an angle $\theta$ to the magnetic field $\mathbf{B}_{\mathbf{0}}$, which is assumed to be in the $(x, z)$-plane (see Fig. 1). The phase velocity of the oscillations is assumed to be smaller in comparison with the electron and proton thermal velocities. The basic set of fluid equations for the cold oxygen ions is given by

$$
\begin{gathered}
\frac{\partial n_{i}}{\partial t}+\frac{\partial\left(n_{i} v_{i x}\right)}{\partial x}=0 \\
\frac{\partial v_{i x}}{\partial t}+v_{i x} \frac{\partial v_{i x}}{\partial x}=-\frac{e}{m_{i}} \frac{\partial \phi}{\partial x}+\Omega_{i} v_{i y} \sin \theta \\
\frac{\partial v_{i y}}{\partial t}+v_{i x} \frac{\partial v_{i y}}{\partial x}=\Omega_{i} v_{i z} \cos \theta-\Omega_{i} v_{i x} \sin \theta
\end{gathered}
$$

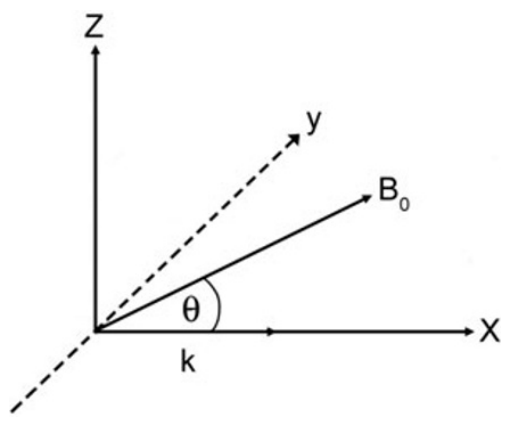

Fig. 1. Geometry of the model.

$$
\frac{\partial v_{i z}}{\partial t}+v_{i x} \frac{\partial v_{i z}}{\partial x}=-\Omega_{i} v_{i y} \cos \theta
$$

where $n_{i}$ is the oxygen ion density, $v_{i x}, v_{i y}$ and $v_{i z}$ are the components of the oxygen ion velocity along $x, y$, and $z$ directions, respectively, $\Omega_{i}\left(=e B_{0} / m_{i}\right)$ is the oxygen ion cyclotron frequency, $m_{i}$ is the oxygen ion mass, $\phi$ is the electrostatic potential of the waves.

The Boltzmann distribution for the electrons and protons, represent respectively as

$$
\begin{gathered}
n_{e}=n_{e 0} \exp \left(\mathrm{e} \phi / T_{e}\right) \\
n_{p}=n_{p 0} \exp \left(-\mathrm{e} \phi / T_{p}\right)
\end{gathered}
$$

where $n_{e 0, p 0}$ and $T_{e, p}$ are the ambient densities and temperatures of the electrons and protons respectively.

Our system of equations is closed with the quasineutrality condition

$$
n_{e}=n_{i}+n_{p}
$$

which is valid for low frequency studies.

In the linear limit, Eqs. (1)-(7) yield the following dispersion relation

$$
\begin{aligned}
\omega^{2}= & \frac{1}{2}\left\{\left(k^{2} C_{s f}^{2}+\Omega_{i}^{2}\right)\right. \\
& \left. \pm\left[\left(k^{2} C_{s f}^{2}+\Omega_{i}^{2}\right)^{2}-4 k^{2} C_{s f}^{2} \Omega_{i}^{2} \cos ^{2} \theta\right]^{1 / 2}\right\}
\end{aligned}
$$

where $\omega$ and $\mathrm{k}$ are the frequency and the wave number, $C_{s f}=\left[n_{i 0} T_{e} T_{p} / m_{i}\left(n_{e 0} T_{p}+n_{p 0} T_{e}\right)\right]^{1 / 2}$ is the effective ion acoustic speed for a three component plasma, and $n_{i 0}$ is the equilibrium value of the oxygen ion number density.

For

$$
4 k^{2} C_{s f}^{2} \Omega_{i}^{2} \cos ^{2} \theta<<\left(k^{2} C_{s f}^{2}+\Omega_{i}^{2}\right)^{2}
$$

we get two modes, namely,

$$
\omega_{+} \approx \Omega_{i}\left(1+k^{2} \rho_{i}^{2}\right)^{1 / 2}
$$

which is the cyclotron mode and

$$
\omega_{-} \approx \frac{k C_{s f} \cos \theta}{\left(1+k^{2} \rho_{i}^{2}\right)^{1 / 2}}
$$

which is the ion-acoustic mode. Here, $\rho_{i}=C_{s f} / \Omega_{i}$ is the effective ion Larmor radius.

We look for solutions of Eqs. (1)-(4) that depend on $\mathrm{x}$ and through a variable $\eta=(x-V t)\left(\Omega_{i} / V\right)$, where $V$ is the phase velocity of the wave. Integrating Eq. (1) and using the conditions $n_{i}=n_{i 0}$ and $v_{x}=v_{0} \cos \theta$ at $\eta=0$ yield

$$
n_{i} v_{x}^{\prime}=-n_{i 0}\left(V-v_{0} \cos \theta\right)
$$

where $v_{x}^{\prime}=v_{x}-V$.

From Eqs. (2), (3) and (4), we get

$$
\begin{aligned}
v_{x}^{\prime} \frac{\partial}{\partial \eta}\left(\frac{v_{x}^{\prime}}{2} \frac{\partial^{2} v_{x}^{\prime 2}}{\partial \eta^{2}}\right. & \left.+\left(\frac{e}{m_{i}}\right) v_{x}^{\prime} \frac{\partial^{2} \phi}{\partial \eta^{2}}\right)+V^{2} v_{x}^{\prime} \frac{\partial v_{x}^{\prime}}{\partial \eta} \\
+ & V^{2} \cos ^{2} \theta\left(\frac{e}{m_{i}}\right) \frac{\partial \phi}{\partial \eta}=0
\end{aligned}
$$


The quasi-neutrality condition gives

$$
\begin{aligned}
v_{x}^{\prime}=-n_{i 0}(V & \left.-v_{0} \cos \theta\right) e^{-\left(e \phi / T_{e}\right)} \\
& \times\left(n_{e 0}-n_{p 0} e^{-\alpha\left(e \phi / T_{e}\right)}\right)^{-1}
\end{aligned}
$$

where $\alpha=\left(T_{e}+T_{p}\right) / T_{p}$.

By combining Eqs. (10) and (11) and introducing the dimensionless quantities $\psi=e \phi / T_{e}, N_{i 0}=n_{i 0} / n_{e 0}, N_{p 0}=$ $n_{p 0} / n_{e 0}, M=V / C_{s}, M_{A}=M-\delta, \delta=v_{0} \cos \theta / C_{s}$, where $C_{s}=\left(T_{e} / m_{i}\right)^{1 / 2}$ is the oxygen ion acoustic speed (in a two component electron-oxygen ion plasma), we obtain

$$
\begin{gathered}
\frac{d}{d \eta}\left[\frac{e^{-\psi}}{\left(1-N_{p 0} e^{-\alpha \psi}\right)} \frac{d^{2} \psi}{d \eta^{2}}+\frac{N_{i 0}^{2} M_{A}^{2} e^{-\psi}}{2\left(1-N_{p 0} e^{-\alpha \psi}\right)} \times\right. \\
\left.\frac{d^{2}}{d \eta^{2}}\left(\frac{e^{-2 \psi}}{\left(1-N_{p 0} e^{-\alpha \psi}\right)^{2}}\right)\right] \\
=-\frac{M^{2} \cos ^{2} \theta e^{\psi}\left(1-N_{p 0} e^{-\alpha \psi}\right)}{\frac{d \psi}{d \eta}} \\
-M^{2} \frac{d}{d \eta}\left[\frac{N^{2} M_{A}^{2}}{\left(1-N_{p 0} e^{-\alpha \psi}\right)}\right]
\end{gathered}
$$

Integrating Eq. (15) and using the conditions $\psi=0$, $d \psi / d \eta=E_{0}$ and $d^{2} \psi / d \eta^{2}=0$ at $\eta=0$, we obtain the following nonlinear wave equation for the normalized electrostatic potential, $\psi$

$$
\chi_{1}(\psi) \frac{d^{2} \psi}{d \eta^{2}}-\chi_{2}(\psi)\left(\frac{d \psi}{d \eta}\right)^{2}+\chi_{3}(\psi)=0
$$

where

$$
\begin{aligned}
& \chi_{1}(\psi)=\frac{N_{i 0}^{2} e^{-2 \psi}}{\left(1-N_{p 0} e^{-\alpha \psi}\right)^{2}}\left\{1+\frac{\alpha N_{p 0} e^{-\alpha \psi}}{\left(1-N_{p 0} e^{-\alpha \psi}\right)}\right\} \\
& -\frac{1}{M_{A}^{2}} \\
& \chi_{2}(\psi)=\frac{N_{i 0}^{2} e^{-2 \psi}}{\left(1-N_{p 0} e^{(-\alpha \psi)}\right)^{2}}\left[2+\frac{\alpha(\alpha+4) N_{p 0} e^{-\alpha \psi}}{\left(1-N_{p 0} e^{-\alpha \psi}\right)}\right. \\
& \left.+\frac{3 \alpha^{2} N_{p 0}^{2} e^{-2 \alpha \psi}}{\left(1-N_{p 0} e^{-\alpha \psi}\right)^{2}}\right] \\
& \chi_{3}(\psi)=\frac{e^{\psi}\left(1-N_{p 0} e^{-\alpha \psi}\right)}{\left(1-N_{p 0}\right)}\left[\frac{M^{2}}{M_{A}^{2}}+E_{0}^{2}\{2\right. \\
& \left.\left.+\frac{\alpha(\alpha+4) N_{p 0}}{\left(1-N_{p 0}\right)}+\frac{3 \alpha^{2} N_{p 0}^{2}}{\left(1-N_{p 0}\right)^{2}}\right\}\right]-\frac{M^{2}}{M_{A}^{2}} \\
& -\frac{M^{2} \cos ^{2} \theta e^{\psi}\left(1-N_{p 0} e^{-\alpha \psi}\right)}{(1-\alpha) N_{i 0}^{2} M_{A}^{4}} \times \\
& {\left[e^{\psi}\left(1-\alpha-N_{p 0} e^{-\alpha \psi}\right)-\left(1-\alpha-N_{p 0}\right)\right]}
\end{aligned}
$$

Equation (16) describes the nonlinear evolution of both ion-cyclotron and ion-acoustic waves and $d \psi / d \eta$ is the normalized electric field. If $N_{p 0}=0$, then Eq. (16) reduces to Eq. (10) of Reddy et al. (2002).

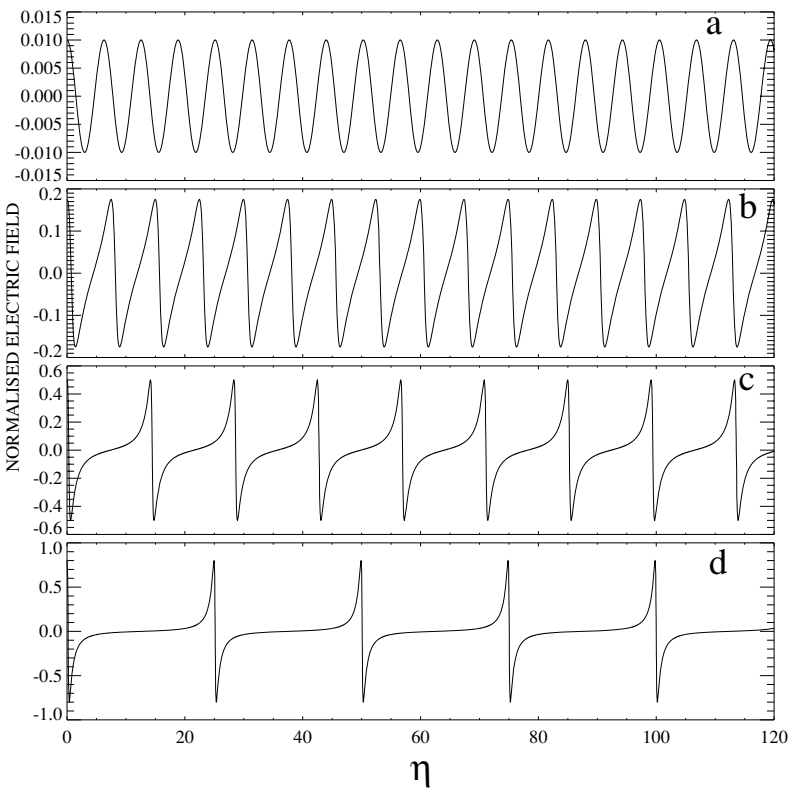

Fig. 2. Numerical solution of the normalized parallel electric field $(=\partial \psi / \partial \eta)$ of nonlinear electrostatic ion cyclotron and ion acoustic waves for the parameters $\theta=2^{\circ}, M=1.25, \delta=0, N_{i 0}=0.75$, $T_{e} / T_{p}=0.1$ and $E_{0}=0.01(\mathrm{a}), 0.175(\mathrm{~b}), 0.50(\mathrm{c})$, and $0.80(\mathrm{~d})$.

\section{Numerical Results and Discussion}

We have numerically solved the nonlinear evolution equation (16) using the Runge-Kutta-Gill method for periodic wave solutions of the electric field $\mathbf{E}(=-(\partial \psi / \partial \eta) \mathbf{x})$, for the plasma parameters encountered on the auroral field lines.

Figure 2 shows the variation of the parallel electric field for $\mathrm{M}=1.25$ and for different driver strengths. For small $E_{0}$, the $E_{\|}$shows ion cyclotron oscillations with a period $\Delta \eta \cong 6.3$ or $\Delta t \cong \tau_{c i}$, where $\tau_{c i}=2 \pi / \Omega_{i}$ is the ion cyclotron period. With increasing driver strength the oscillations become nonlinear with increasing periodicity. For sufficiently large driver amplitude $\left(E_{0} \geq 0.8\right)$, one obtains the driven ion acoustic wave which is highly spiky, with a period $\sim 4.0 \tau_{c i}$. This behaviour is observed to be consistent with the measurements for $E_{\|}$by the FAST satellite in figure 6 of Ergun et al. (1998).

The variation of normalized electric field with oxygen ion density is shown in Fig. 3. The period of oscillations increases with decreasing $N_{i 0}$. For large $N_{i 0}, E_{\|}$exhibits the oscillations in the ion cyclotron period range and the waveform of these oscillations tends to be of the sawtooth type. For sufficiently small oxygen ion density $\left(N_{i 0}=0.15\right)$, we get a highly spiky waveform of driven ion acoustic mode.

Figure 4 shows the variation of the parallel electric field for $E_{0}=0.5, M=1.25$ and for different oxygen beam velocities. Here we found that the period of the spiky structures decrease as the beam velocity varies from -0.2 to 0.2 .

) The oxygen ion flow parallel to $\mathbf{B}_{\mathbf{0}}$ would decrease the period of oscillations, whereas the antiparallel flow increases the period of the spiky structures.

The effect of Mach number, $M$ is shown in Fig. 5. We have ion cyclotron oscillations for $M \leq 1.0$. As the Mach number increases, the waveform become more steeper but 


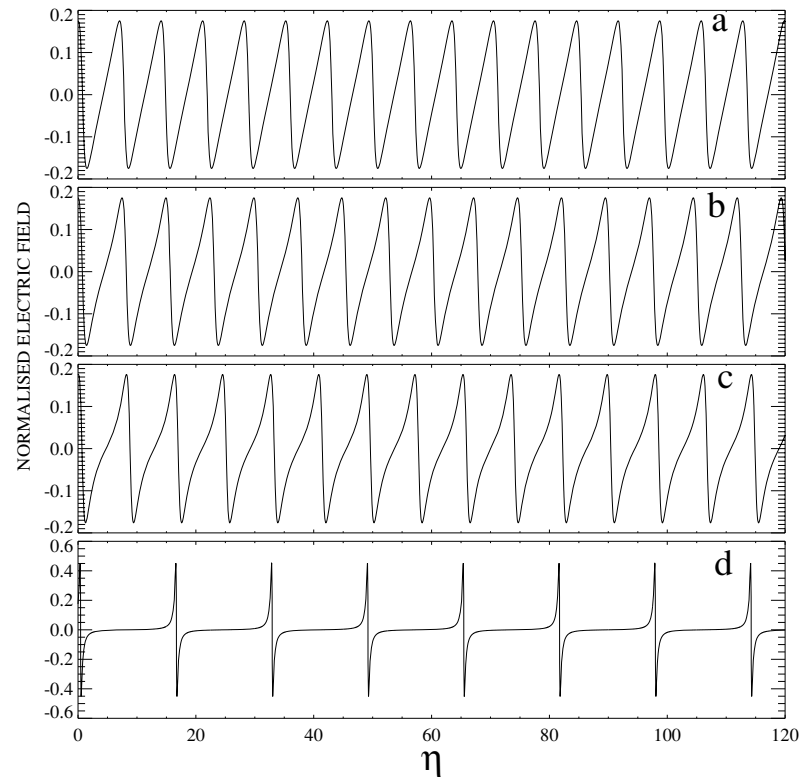

Fig. 3. Variation of the normalized parallel electric field for $E_{0}=0.175$ and $N_{i 0}=1.0$ (a), 0.75 (b), 0.55 (c) and 0.15 (d). All other parameters are same as in Fig. 2.

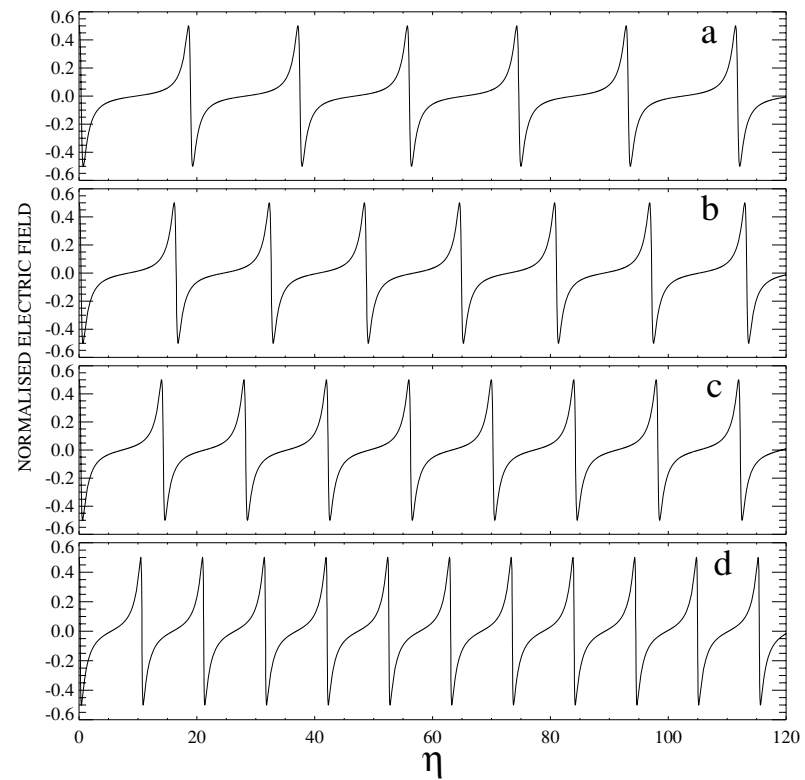

Fig. 4. The normalized parallel electric field for $E_{0}=0.5$ and $\delta=-0.2$ (a), -0.1 (b), 0.0 (c) and 0.2 (d). All other parameters are same as in Fig. 2.

the period of the oscillations remain the same. For $M \geq 1.0$, we get the driven ion acoustic mode which is highly spiky and the period decreases with increasing Mach number.

In Fig. 6, we have varied the propagation angle $\theta$. The other fixed parameters are $M=1.25, E_{0}=0.2, N_{p 0}=$ $0.25, T_{e} / T_{p}=0.1$, and $\delta=0.0$. The period of the oscillations decreases with increasing propagation angle. The oscillations are of sawtooth type and the nature remains same as the propagation angle varies. For large $\theta$, we obtain ion cyclotron oscillations.

Figure 7 displays the variation of the temperature ratio $T_{e} / T_{p}$. Here we observed that the period of the oscillations

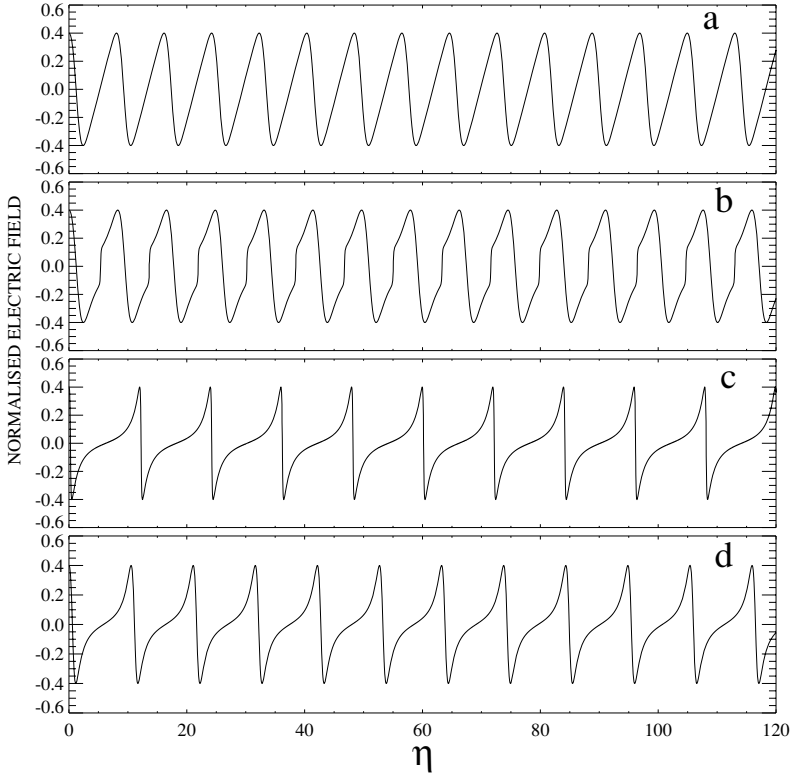

Fig. 5. The normalized parallel electric field for $E_{0}=0.4$ and $M=0.1$ (a), 0.1705 (b), 1.05 (c) and 4.5 (d). All other parameters are same as in Fig. 2.

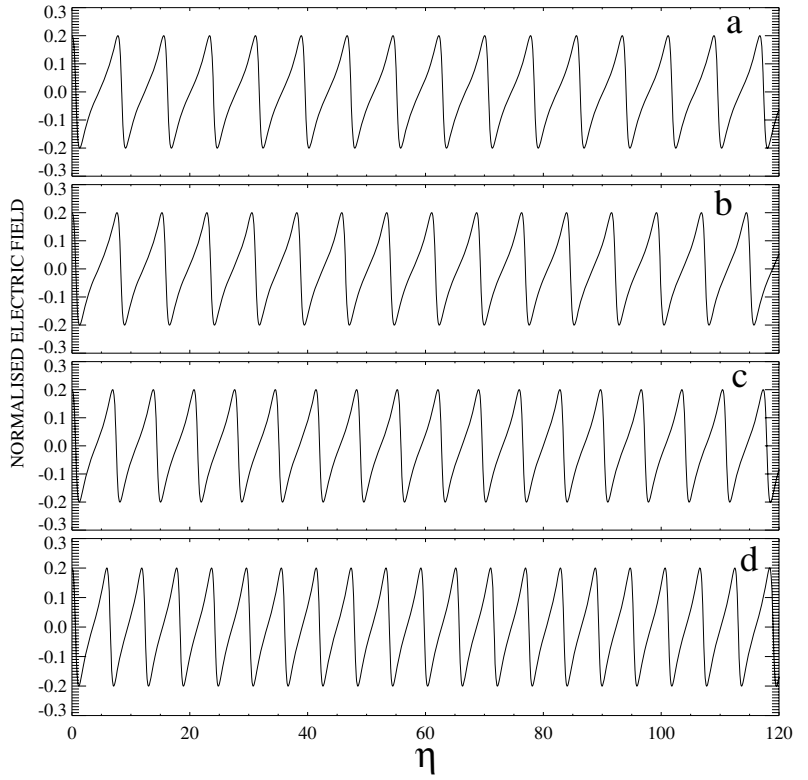

Fig. 6. The normalized parallel electric field for $E_{0}=0.2$ and $\theta=2^{\circ}$ (a), $15^{\circ}$ (b), $40^{\circ}$ (c) and $85^{\circ}$ (d). All other parameters are same as in Fig. 2.

increases with increasing $T_{e} / T_{p}$. When the ratio $T_{e} / T_{p}$ is small we have a sawtooth type waveform. For higher $T_{e} / T_{p}$, we obtain the driven ion acoustic mode and the waveform becomes highly spiky.

\section{Conclusions}

We have studied the nonlinear evolution of low frequency electrostatic structures arising from a coupling of the oxygen ion cyclotron and oxygen ion acoustic waves for any direction of propagation with respect to the ambient magnetic field, in the presence of hot protons. Past studies (Reddy et al., 2002; Bharuthram et al., 2002) revealed spiky electric field structures which could explain the FAST satellite 


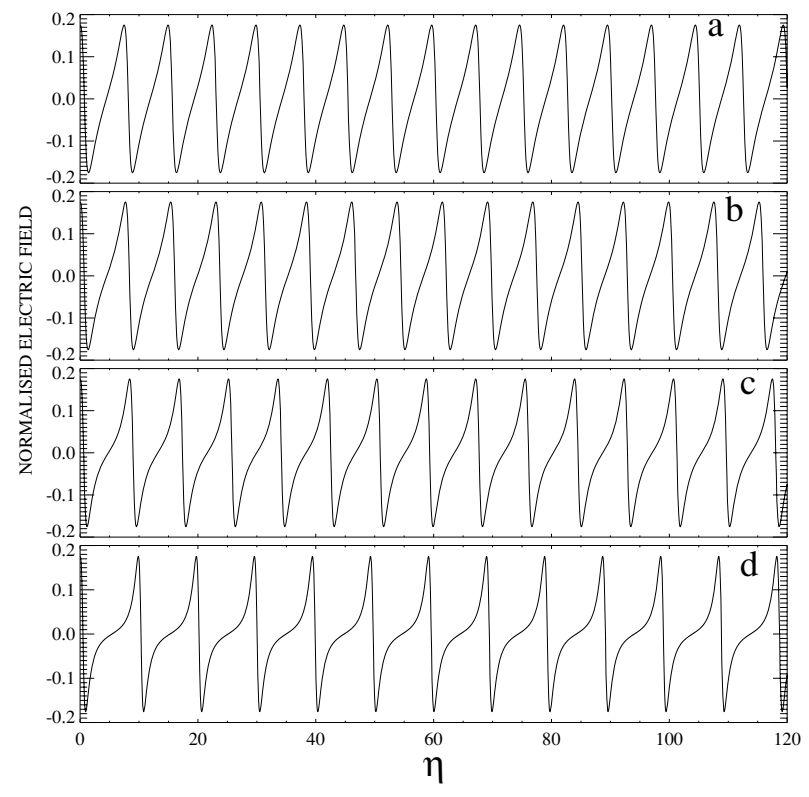

Fig. 7. The normalized parallel electric field for $E_{0}=0.175$ and $T_{e} / T_{p}=0.1$ (a), 0.5 (b), 1.5 (c) and 3.0 (d). All other parameters are same as in Fig. 2.

observations of the strong spiky waveforms in the parallel electric field in association with ion cyclotron oscillations in the perpendicular electric fields.

The inclusion of oxygen ions has significant effect on the evolution of nonlinear electric field structures. The model exhibits ion cyclotron oscillations when the oxygen ion density is high. The driven ion acoustic mode exists only for sufficiently low oxygen ion density and also the waveform is highly spiky. In this paper the nonlinear mode coupling occurs in the presence of a driver, i.e., it is a driven process. It is assumed that both the ion cyclotron and ion acoustic modes propagate in the same direction. We do not consider the coupling between the modes propagating at different angles to the magnetic field. This process involves frequency and wave number matching, and represents an higher order wave-wave coupling, which is beyond the scope of this paper.

We have shown the waveforms depends sensitively on the plasma parameters, and that the observed waveforms could be produced while the background plasma parameters are changing within some limits. It is an interesting result. As to how a change in a particular parameter affect the waveform, is a complex issue as it involves the nonlinear interaction between the cyclotron and acoustic mode in the presence of a driver field. We are trying to understand the underlying physics.

It is found that spiky structures can exists over a wide angle of wave propagation. For nearly parallel angle of propagation, the parallel electric fields dominate over the perpendicular electric fields. Mixtures of parallel and obliquely propagating nonlinear waves could provide a natural explanation for the spikiness in the parallel and perpendicular electric fields components. For nearly parallel propagation, $E_{\|}$shows ion cyclotron oscillations when the driver strength is low, whereas for a stronger driver strength we obtain highly spiky driven ion acoustic waves with a period $4.0 \tau_{c i}$. From Figs. 2-7 for the auroral plasma parameters, and $T_{e}=10-100 \mathrm{eV}, B_{0}=1300 \mathrm{nT}$, we get $E_{\|} \sim(50-250)$ $\mathrm{mV} / \mathrm{m}$. These results may be relevant to the observations of nonlinear cyclotron waves with amplitudes of the order of $200 \mathrm{mV} / \mathrm{m}$ or more by Cattell et al. (1998).

The observations in the auroral region (Ergun et al., 1998) show the multi-harmonic low frequency waves which are associated with coherent spiky electrostatic potential and electric field structures. This would require the inclusion of ion kinetic effects in the model, which is beyond the scope of this paper. This situation will be addressed in the future paper.

In this paper, we have considered electrons and protons with Boltzmann distributions. These approximations can be justified provided the phase velocity $\left(V_{p h}\right)$ is much smaller than the electron $\left(V_{t e}\right)$ and proton $\left(V_{t p}\right)$ thermal speed respectively. Using Eq. (11), we find that

$$
\frac{V_{p h}{ }^{2}}{V_{t p}{ }^{2}} \approx \frac{m_{p} n_{i 0}}{m_{i} n_{e 0}}\left[\frac{T_{e} / T_{p}}{1+\frac{n_{p 0} T_{e}}{n_{e 0} T_{p}}}\right]
$$

and

$$
\frac{V_{p h}{ }^{2}}{V_{t e}{ }^{2}} \approx \frac{m_{e} n_{i 0}}{m_{i} n_{e 0}}\left[\frac{1}{1+\frac{n_{p 0} T_{e}}{n_{e 0} T_{p}}}\right]
$$

These conditions are satisfied for all the numerical parameters considered in this paper. We give here the estimates for the largest values of $V_{p h} / V_{t p}$ and $V_{p h} / V_{t e}$ (the worst case) which corresponds to the parameters of Fig. 7, i.e., $T_{e} / T_{p}=3.0$ and $N_{i 0}=0.75$. For this we have the ratio, $V_{p h} / V_{t p} \approx 0.28$, and $V_{p h} / V_{t e} \approx 0.015$, which are less than one and justifies our approximation for the Boltzmann distributions for electrons and protons.

The present analysis can be extended to include the effects due to warm oxygen ions, charge separation, and multi-beams to make it closer to the observations.

Acknowledgments. We acknowledge support from the NRF, South Africa under the India-South Africa Bilateral Programme. GSL would like to thank Council of Scientific and Industrial Research, Government of India for support under the Emeritus Scientist Scheme.

\section{References}

Andre, M., H. Koskinen, G. Gustafsson, and R. Lundin, Ion waves and upgoing ion beams observerd by the Viking satellite, Geophys. Res. Lett., 14, 463-466, 1987.

Bharuthram, R., R. V. Reddy, G. S. Lakhina, and N. Singh, Low-frequency nonlinear waves in the auroral plasma, Physica Scripta, T98, 137-140, 2002.

Cattell, C., R. Bergmann, K. Sigsbee, C. Carlson, C. Chaston, R. Ergun, J. McFadden, F. S. Mozer, M. Temerin, R. Strangeway, R. Elphic, L. Kistler, E. Moebius, L. Tang, D. Klumper, and R. Pfaff, The associatation of electrostatic ion cyclotron waves, ion and electron beams and filed-aligned current: FAST observations of an auroral zone crossing near midnight, Geophys. Res. Lett., 25, 2053-2056, 1998

Ergun, R. E., C. W. Carlson, J. P. McFadden, F. S. Mozer, G. T. Delory, W. Peria, C. C. Chaston, M. Temerin, R. Elphic, R. Strangeway, R. Pfaff, C. A. Cattell, D. Klumpar, E. Shelley, W. Peterson, E. Moebius, and L. Kistler, FAST satellite observations of electric field structures in the auroral zone, Geophys. Res. Lett., 25, 2025-2028, 1998. 
Ganguli, G., Slinker, V. Gavrishchaka, and W. Scales, Low-frequency oscillations in a plasma with spatially variable field-aligned flow, Phys. Plasmas, 9, 2321-2329, 2002.

Kelley, M. C., E. A. Bering, and F. S. Mozer, Evidence that the electrostatic ion cyclotron instabilty is saturated by ion heating, Phys. Fluids, $\mathbf{1 8}$, 1590-1591, 1975.

Kim, S-H., R. L. Merlino, and G. I. Ganguli, Generation of spiky potential structures associated with multi-harmonic electrostatic ion cyclotron waves, Preprint, 2005.

Kindel, J. M. and C. F. Kennel, Topside current instabilities, J. Geophys. Res., 76, 3055-3078, 1971 .

Moolla, S., R. Bharuthram, S. V. Singh, and G. S. Lakhina, Nonlinear high frequency waves in the magnetosphere, Pramana-J. Phys., 61, 1209$1214,2003$.

Mozer, F. S., C. W. Carlson, M. K. Hudson, R. B. Torbert, B. Parady, J.
Yatteau, and M. C. Kelley, Observations of paired electrostatic shocks in the polar magnetosphere, Phys. Rev. Lett., 38, 292-295, 1977.

Mozer, F. S., R. Ergun, M. Temerin, C. Cattell, J. Dombeck, and J. Wygant, New features of time domain electric-field structures in the auroral acceleration region, Phys. Rev. Lett., 79, 1281-1284, 1997.

Reddy, R. V., G. S. Lakhina, N. Singh, and R. Bharuthram, Spiky parallel electrostatic ion cyclotron and ion acoustic waves, Nonlinear Proc. Geophys., 9, 25-29, 2002.

Temerin, M., M. Woldorff, and F. S. Mozer, Nonlinear steepening of the electrostatic ion cyclotron wave, Phys. Rev. Lett., 43, 1941-1943, 1979.

R. V. Reddy (e-mail: vreddy@iigs.iigm.res.in), S. V. Singh, G. S. Lakhina, and R. Bharuthram 